There are no routine outcome measures to judge the effectiveness of this NICE guidance. The process measures above have demonstrated variable implementation in GUM services in England. With present resources, as one example providing 15-20 min of one-to-one structured discussions based on behavioural change theories is not deliverable. Service commissioners should ensure that services are implementing guidance and using recognised standards for the management of sexually transmitted infections. Prevention of sexually transmitted infections should be targeted, informed by the sexual health profile of local populations with resources identified by commissioners for prevention activities as integral to service delivery.

\section{P5-S6.39 INTEGRATING MEDICATION ASSISTED THERAPY WITH BUPRENORPHINE AND OTHER HARM REDUCTION INTERVENTIONS INTO AN URBAN PUBLIC HEALTH DEPARTMENT STD CLINIC IN THE SOUTHWESTERN USA}

doi:10.1136/sextrans-2011-050108.595

${ }^{1} \mathrm{~B}$ Trigg, ${ }^{2} \mathrm{M}$ Murphy, ${ }^{3} \mathrm{~A}$ Tsang. ${ }^{1}$ New Mexico Department of Health (retired), Albuquerque, USA; ${ }^{2}$ Stanford Buprenorphine Induction Program, Albuquerque, USA; ${ }^{3} \mathrm{CDC}$ Associate, Albuquerque, USA

Background Public Health Department STD clinics often serve high risk populations that have limited access to treatment of opiate addiction using evidence-based medication assisted therapies (buprenorphine and methadone) and to other clinical preventive services. In Albuquerque, New Mexico, in the southwestern US, an urban STD clinic has developed an integrated buprenorphine induction program for injecting heroin users that prioritises those recently released from a county jail. Patients are also recruited directly from the STD clinic and from a co-located syringe exchange program.

Methods Patients enrolled in the buprenorphine induction program who met specific screening criteria were tested for chlamydia, gonorrhoea, HIV, hepatitis C and syphilis. Patients who completed 2 months of buprenorphine were referred to primary care providers for continuation of treatment.

Results In the first 2 years, a total of 291 patients received buprenorphine. 85 clients $(29.2 \%)$ were referred to the program from public health programs including STD clinic, family planning and harm reduction. 67 (23.0\%) were recently released from jail. Of the first 191 patients, 55 (28\%) completed 2 months of buprenorphine treatment. 1 of 160 clients screened for syphilis were positive. 68 of 131 (51.9\%) screened for hepatitis C were positive. 10 of 89 (11.2\%) screened for gonorrhoea and chlamydia were positive for one or both infections. 194 were tested for HIV; none were positive.

Conclusion An integrated STD clinic and opiate addiction treatment program can provide comprehensive services to a high risk population.

\section{Health services and policy poster session 7: screening

P5-S7.01 EXPLORING THE ACCEPTABILITY OF MEDICAL,
EDUCATIONAL AND SPORT SETTINGS FOR STI
SCREENING: STRATIFIED RANDOM PROBABILITY SURVEY
OF YOUNG MEN IN THE UK

doi:10.1136/sextrans-2011-050108.596

${ }^{1} \mathrm{C}$ Estcourt, ${ }^{1} \mathrm{~J}$ Saunders, ${ }^{2} \mathrm{C}$ Mercer, ${ }^{1} \mathrm{~L}$ Sutcliffe, ${ }^{2} \mathrm{G}$ Hart. ${ }^{1}$ Queen Mary University of London, London, UK; ${ }^{2}$ University College London, London UK

Background UK prevalence of STIs in young people is rising. Although a similar number of infections are diagnosed in men and women, men account for only $20 \%$ of National Chlamydia Screening Program tests. New strategies are required to increase uptake of STI screening in young men.

Methods Stratified random probability survey of men (18-35 years) using computer-assisted personal- and self-interviews, including use of healthcare; sporting activity; acceptability of accessing urine/oral fluid self-taken tests for STIs and HIV in a variety of healthcare and non-healthcare settings.

Results Data were collected from 411 (632 weighted) men median age 28 y; $39 \%$ aged $<25$ y. $29 \%$ and $20 \%$ of men had previously tested for STIs and HIV, respectively. Two-thirds of men $<25 \mathrm{y}$ had tested in the last year vs one-fifth of men $>25 y(p<0.0001) .75 \%$ of men had seen their Family Physician within the last year. 91\% of men would be willing to self-test for STI/ HIV. Primary Care settings (80\%), sexual health clinics (67\%) and pharmacies $(65 \%)$ were the most acceptable test kit pick-up points. Further education settings were more popular than school settings as pick-up points ( $42 \%$ vs $28 \%$ ) and the workplace was acceptable to $22 \%$ of men. Of the $391(69 \%)$ men who reported playing sport in the last 4 weeks $18 \%$ found a sports club/centre acceptable. Among the 37\% men who reported playing the most popular sport (soccer), $47 \%$ and $43 \%$ said they would be willing to pick-up STI and HIV test kits, respectively, from their club. This did not vary by whether they had previously tested for STI/ HIV.

Conclusions Almost one-third of men under 25 have already tested for STI/ HIV. Most men have seen their Family Physician in the last year, which challenges the assumption that young men infrequently attend primary care. Primary care is a highly acceptable setting for STI/ HIV screening. Non-traditional settings such as soccer clubs are acceptable to some men but further research is needed to better understand the barriers and opportunities with this approach to testing.

\section{P5-S7.02 TRENDS IN SCREENING FEMALES FOR CHLAMYDIA IN JUVENILE DETENTION CENTERS US 2005-2009}

doi:10.1136/sextrans-2011-050108.597

${ }^{1,2} \mathrm{D}$ R Newman, ${ }^{1,2} \mathrm{~T}$ A Peterman, ${ }^{1,2} \mathrm{D}$ E Collins. ${ }^{1}$ Centers for Disease Control and Prevention, Atlanta, Georgia, USA; ${ }^{2}$ Division of STD Prevention, Atlanta, Georgia, USA

Background CDC began development of Performance Measures in 1999 and 58 Project Areas have continuously reported on the measures twice yearly since 2005. Two Performance Measures from Juvenile Detention Centers (screening in 2004 and positivity in 2009) were developed to capture the burden of chlamydia infection in this underserved population of adolescent females.

Objectives To assess changes in performance of screening of females in juvenile detention centers (JDC's) from the US as reported through Performance Measures and to obtain a measure of the positivity detected.

Methods 50 US States, six US cities, Washington, DC and Puerto Rico are asked to report the percentage of females screened for chlamydia twice yearly from all juvenile detention centers that admit 500 or more adolescent females annually. Project Areas with no facilities that admit 500 or more are to report on one or more facilities of their choice. A chlamydia positivity measure was added in 2009. We collapsed the 2 half-yearly reports for each Project Area into yearly summaries from 2005 to 2009.

Results The percentage of Project Areas that were able to report any chlamydia screening from their JDC's increased from 60\% in 2005 to $84 \%$ in 2009 . The mean percentage of Project Areas reporting any screening over the 5 years was $80 \%$. Many Project Areas reported from multiple JDC's during 2005-2009 with California reporting from 12 facilities and Ohio reporting from 11 facilities. The reported percentage of juvenile females screened by year were $54,63,59,60$ and $51 \%$ from 2005 to 2009 . The percentage screened ranged from 
$3 \%$ to $100 \%$. The mean percentage of females screened over the 5 years was $57 \%$. In 2009 , the mean chlamydia positivity for all Project Areas was $13.8 \%$. For facilities $(n=69)$ that screened at least 100 females in 2009, the mean chlamydia positivity detected was $13.7 \%$, with a median of $12.6 \%$ and positivity ranging from 6 to $31 \%$. Almost half (48\%) of the Project Areas reported from 49 facilities for all 10 reporting periods across the 5 -year period. The percentage screened in these 49 facilities increased 59, 64, 66, 69 and $69 \%$ from 2005 to 2009 . The mean per cent positivity for these facilities in 2009 was $12.5 \%$.

Conclusions Juvenile Detention Centers are excellent venues from which to detect chlamydia infections in adolescent females. Additional efforts are needed to increase the number of facilities that routinely offer screening and increase the proportion screened.

\section{P5-S7.03 SYPHILIS SCREENING FOR HIGH-RISK GROUPS IN A LARGE-SCALE HIV PREVENTION PROGRAM IN INDIA: UPTAKE AND TRENDS}

doi:10.1136/sextrans-2011-050108.598

${ }^{1} \mathrm{M}$ Parthasarathy, ${ }^{1} \mathrm{~N}$ Potty, ${ }^{1} \mathrm{~A}$ Gurung, ${ }^{1} \mathrm{~A}$ Das, ${ }^{2} \mathrm{P}$ Parimi. ${ }^{1}$ Family Health International, Chennai, India; ${ }^{2}$ Alliance, Hyderabad, India

Background Avahan, a large-scale HIV prevention program in India, provides syphilis screening as a part of its service package for highrisk groups (HRGs) including female sex workers, men who have sex with men (MSM) and injecting drug users through its targeted intervention clinics. The objective of this retrospective study was to understand the uptake and trends in reactivity patterns of syphilis screening tests at Avahan clinics from 2005 to 2009.

Methods Syphilis screening was done with either Rapid Plasma Reagin (RPR test, provided on-site or through referrals to local laboratories) or a point-of-care immunochromatographic strip test (ICST) using whole blood. ICST was introduced to the program in 2007. The clinical records of the HRGs attending Avahan clinics in six high HIV prevalence states in India from January 2005 to December 2009 were collated in an "individual tracking sheet" database and analysed with STATA software version 10.

Results The overall proportion of clinic attendees screened for syphilis increased consistently during the study period from $2.6 \%$ in 2005 to $21 \%$ in 2009 . The increasing trend in uptake and actual proportions were similar in all risk groups. The use of ICST as a screening test increased from $8 \%$ in 2007 to $70 \%$ in 2009 thus becoming the predominant screening method. During this period, the uptake of syphilis screening also increased from $9 \%$ in 2007 to $21 \%$ in 2009 see Abstract P5-S7.03 table 1. A declining trend was found with the overall syphilis sero-reactivity rates (ICST and RPR), from 7\% in 2006 to $4 \%$ in 2009 and this decreasing trend was found in all risk groups. The proportion of active high-titre syphilis $(>1: 8)$ to all reactive RPR tests (any titre) declined from 61\% in 2005 to $30 \%$ in 2009.

Conclusions The improved uptake of syphilis screening has lead to improved detection and appropriate management of cases of latent syphilis. The results show that introduction of rapid point-of-care tests likely contributed to the improved uptake of syphilis screening.

Abstract P5-S7.03 Table 1 Uptake of syphilis screening by RPR and ICST

\begin{tabular}{lcclc}
\hline Year & $\begin{array}{l}\text { Total individuals } \\
\text { attending clinics }\end{array}$ & $\begin{array}{l}\text { \# Screened } \\
\text { for syphilis (\%) }\end{array}$ & $\begin{array}{l}\text { Proportion } \\
\text { screened } \\
\text { with RPR (\%) }\end{array}$ & $\begin{array}{l}\text { Proportion } \\
\text { screened } \\
\text { with ICST (\%) }\end{array}$ \\
\hline 2005 & 43394 & $1112(2.6 \%)$ & 100 & 0 \\
2006 & 108836 & $5700(5.2 \%)$ & 100 & 0 \\
2007 & 169612 & $15179(8.9 \%)$ & 91.8 & 8.2 \\
2008 & 220877 & $28502(12.9 \%)$ & 60.1 & 39.9 \\
2009 & 286991 & $60412(21.1 \%)$ & 30.8 & 69.2 \\
\hline
\end{tabular}

Rapid tests may be considered for syphilis screening at other resource-constrained primary care sites in India such as targeted intervention and ante-natal clinics.

\section{P5-S7.04 WHO PARTICIPATES IN THE DUTCH CHLAMYDIA SCREENING? A STUDY ON DEMOGRAPHIC AND BEHAVIOURAL CORRELATES OF (REPEATED) PARTICIPATION AND POSITIVITY}

doi:10.1136/sextrans-2011-050108.599

${ }^{1} \mathrm{E} O$ de Coul, ${ }^{2} \mathrm{H}$ Götz, ${ }^{3} \mathrm{~J}$ Van Bergen, ${ }^{4} \mathrm{E}$ Brouwers, ${ }^{5} \mathrm{H}$ Fennema, ${ }^{4} \mathrm{C}$ Hoebe, ${ }^{5} \mathrm{R}$ Koekenbier, ${ }^{2} \mathrm{~S}$ van Ravesteijn, ${ }^{3} \mathrm{~L}$ Pars, ${ }^{1} \mathrm{I}$ van den Broek. ${ }^{1} R I V M$, Bilthoven, Netherlands; ${ }^{2} G G D$ Rotterdam-Rijnmond, Netherlands; ${ }^{3}$ SOA AIDS Nederland, Netherlands; ${ }^{4} G G D$ Oosterlijk Zuid-Limburg, Netherlands; ${ }^{5} G G D$ Amsterdam, Netherlands

Background Achieving adequate levels of participation and capturing high risk groups are key stones for the effectiveness of large-scale Chlamydia screening programs. We examined the determinants of (repeated) participation and Chlamydia positivity to evaluate to what extent high risk groups were reached in a large scale Internet-based screening program in the Netherlands.

Methods The Chlamydia Screening Implementation was initiated in three regions among people aged 16-29 years. Data from the first two screening rounds (2008-2010), in which approximately 280000 persons were invited annually, were analysed. Socio-demographic and behavioural correlates of screening participation and positivity were studied in multilevel logistic regression models. Cluster was added as a second level of analysis, taking into account the effect of the neighbourhood-based invitations (to cover social or sexual networks).

Results The same socio-demographic factors associated with lower screening uptake were also associated with higher Ct-positivity such as young age, non-Dutch origin, lower education, high community risk, low SES, in round 1 as well as 2 . At the same time, behavioural risk factors such as having casual partners, $\geq 2$ partners in $<6$ months, concurrent partners, and a history of STI, were associated with higher participation. A small cluster effect for screening uptake was observed, independent from community risk and individual risk factors. The model for repeated participation showed that men, Turkish/Moroccans and persons $\leq 20$ years were less likely to participate twice, while people having a short-term relationship, a non-Dutch partner or concurrent partners were more likely to participate again. Ct-positives, who did not participate in the rescreening after 6 months, were also less likely to participate in the second screening round.

Conclusions Socio-demographic factors associated with lower participation were also associated with higher Ct-positivity, showing that, high-risk demographic groups were more difficult to mobilise than low-risk groups. However, independent of this, higher behavioural risk levels were associated with higher participation rates (especially in the model for repeated participation), suggesting self-selection for screening based on the persons' risk (perception) in both low and high community risk groups. Our study shows the complexity of the process-including individual as well as community factors and their interaction-as to whether or not be screened for chlamydia.

\section{P5-S7.05 CHLAMYDIA SCREENING IN AN INTERNATIONAL RESORT COMMUNITY: AN OUTREACH PROGRAM TO EXPAND ACCESS}

doi:10.1136/sextrans-2011-050108.600

${ }^{1} \mathrm{M}$ Collins, ${ }^{2} \mathrm{R}$ Holehouse, ${ }^{1} \mathrm{~J}$ Kaczorowski. ${ }^{1}$ University of British Columbia, Whistler, Canada; ${ }^{2}$ Options for Sexual Health BC, Canada

Objective To evaluate an event-based outreach Chlamydia (CT) screening program pilot developed to address barriers to access in a 